\title{
Ocean Color Instrument Integration and Testing
}

\author{
Susanna Petro \\ NASA/Goddard Space Flight Center \\ 8800 Greenbelt Road \\ Greenbelt, MD 20771 \\ 301-286-2039 \\ susanna.petro-1@nasa.gov \\ George Hilton \\ Newton Engineering And Product Development \\ Work performed for NASA/Goddard Space Flight Center \\ 8800 Greenbelt Road \\ Greenbelt, MD 20771 \\ 301-286-3759 \\ george.m.hilton@nasa.gov
}

\author{
David Sohl \\ NASA/Goddard Space Flight Center \\ 8800 Greenbelt Road \\ Greenbelt, MD 20771 \\ 301-286-1055 \\ david.w.sohl@nasa.gov \\ Mellina Espiritu \\ NASA/Goddard Space Flight Center \\ 8800 Greenbelt Road \\ Greenbelt, MD 20771 \\ 301-286-3906 \\ ana.mellina.p.espiritu@nasa.gov
}

Abstract -This paper describes the plans, flows, key facilities, components and equipment necessary to fully integrate, functionally test, qualify and calibrate the Ocean Color Instrument (OCI) on the Plankton, Aerosols, Clouds, and oceans Ecosystem (PACE) observatory. PACE is currently in the design phase of mission development. It is scheduled to launch in 2022, extending and improving NASA's twenty-year record of satellite observations of global ocean biology, aerosols and clouds. PACE will advance the assessment of ocean health by measuring the distribution of phytoplankton, which are small plants and algae that sustain the marine food web. It will also continue systematic records of key atmospheric variables associated with air quality and the Earth's climate. PACE's primary sensor, the OCI, is a highly advanced optical spectrometer that will be used to measure properties of light over portions of the electromagnetic spectrum. It will enable continuous measurement of light at finer wavelength resolution than previous NASA satellite sensors, extending key system ocean color data records for climate studies. The color of the ocean is determined by the interaction of sunlight with substances or particles present in seawater such as chlorophyll. By monitoring global phytoplankton distribution and abundance with unprecedented detail, the OCI will contribute to a better understanding of the complex systems that drive ocean ecology and it's impacts on global fisheries. This paper will focus on the Integration and Test (I\&T) activities for OCI while it is at the NASA Goddard Space Flight Center. The OCI integration consists of assembly and alignment of the rotating telescope, electronics box integration, fixed deck assembly integration, thermal systems integration and the final assembly and testing. This I\&T phase will be followed by the OCI calibration and characterization, environmental tests which include electromagnetic interference (EMI)/electromagnetic compatibility (EMC), vibration with sine sweep, acoustics, shock, thermal balance, thermal vacuum, mass properties and center of gravity. This paper will briefly discuss OCI shipment and delivery to the spacecraft vendor for observatory level $I \& T$ as well as some launch preparation activities
Table of Contents

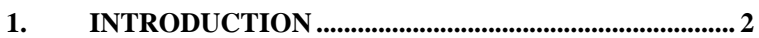

1.1 PACE MISSION OVERVIEW ................................................. 2

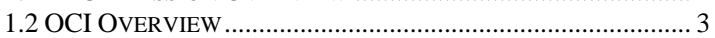

2. INTEGRATION AND TEST GROUND SUPPORT EQUIPMENT .............................................................................. 5

3. OCI I\&T GROUND SYSTEM........................................... 5

3.1 INTEGRATED TEST AND OPERATIONS SYSTEM (ITOS) ........ 5

3.2 FRONT END DATA SYSTEM (FEDS) ……………………..... 6

3.3 REAL-TIME SCIENCE PROCESSING WORKSTATION.............. 6

3.4 OCI INTEGRATED TRENDING AND PLOTTING SYSTEM (ITPS)

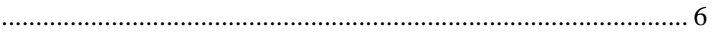

4. OCI INSTRUMENT INTEGRATION AND

CALIBRATION . .6

5. OCI INSTRUMENT CALIBRATION CHALLENGES

DURING I\&T.........................................................................

5.1 CALIBRATION SOURCES ........................................................ 7

5.2 LINE SPREAD FUNCTION (LSF) BENCH ................................ 9

5.3 STRAY-LIGHT …………………………………............ 9

5.4 SNAPSHOT MODE AND TELESCOPE ROTATION...................... 9

5.5 POLARIZATION SENSITIVITY .............................................. 10

6. OCI INSTRUMENT ENVIRONMENTAL TESTING10

6.1 Thermal Balance/Thermal VACUUM TeST ................. 10

6.2 EMC/EMI .................................................................... 12

6.3 VIBRATION TEST …………………………………....... 12

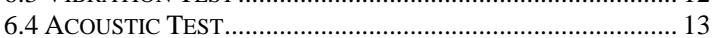

6.5 MASS PROPERTIES AND CG MEASUREMENT....................... 13

7. OCI INSTRUMENT DELIVERY AND

OBSERVATORY I\&T ACTIVITIES

8. OCI INSTRUMENT LAUNCH SITE ACTIVITIES.. 14

9. SUMMARY ….................................................................. 14

10. REFERENCES .................................................. 14

11. BIOGRAPHY............................................................. 15 


\section{INTRODUCTION}

\subsection{PACE Mission Overview}

The Plankton, Aerosol, Cloud, ocean Ecosystem (PACE) mission is a strategic climate continuity mission that was defined in the 2010 document Responding to the Challenge of Climate and Environmental Change: NASA's Plan for ClimateCentric Architecture for Earth Observations and Applications from Space (referred to as the "Climate Initiative”) [1]. The Climate Initiative complements the National Aeronautics and Space Administration (NASA)'s implementation of the National Research Council's Decadal Survey of Earth Science at NASA, at the National Oceanic and Atmospheric Administration (NOAA), and at the United States Geological Survey (USGS), entitled Earth Science and Applications from Space: National Imperatives for the Next Decade and Beyond [2].

PACE will extend the high quality ocean ecological, ocean biogeochemical, cloud, and aerosol particle data records begun by NASA in the 1990s, building on the heritage of the Sea-Viewing Wide Field-of-View Sensor (SeaWiFS), the Moderate Resolution Imaging Spectroradiometer (MODIS), the Multi-angle Imaging SpectroRadiometer (MISR), and the Visible Infrared Imaging Radiometer Suite (VIIRS). The mission will be capable of collecting radiometric and polarimetric measurements of the ocean and atmosphere, from which these biological, biogeochemical, and physical properties will be determined. PACE data products will not only add to existing critical climate and Earth system records, but also answer new and emerging advanced science questions related to Earth's changing climate.

PACE is classified as a Category 2 mission, per the criteria in NASA Procedural Requirement (NPR) 7120.5E, NASA Space Flight Program and Project Management Requirements. The mission classification is C according to NPR 8705.4B, Risk Classification for NASA Payloads.

The PACE observatory is comprised of three instruments, an Ocean Color Instrument (OCI) and two polarimeters, the Hyper-Angular Rainbow Polarimeter 2 (HARP2) and the Spectro-Polarimeter for Exploration (SPEXOne). The OCI is the primary instrument on the observatory and is being developed at Goddard Space Flight Center (GSFC). The OCI is a hyper-spectral scanning (HSS) radiometer designed to measure spectral radiances from the ultraviolet to shortwave infrared (SWIR) to enable advanced ocean color and heritage cloud and aerosol particle science. The HARP2 and SPEXOne are secondary instruments on the PACE observatory, acquired outside of GSFC. The Hyper-Angular Rainbow Polarimeter instrument (HARP2) is a wide swath imaging polarimeter that is capable of characterizing atmospheric aerosols for purposes of sensor atmospheric correction as well as atmospheric science. The SPEXOne provides atmospheric aerosol and cloud data at high temporal and spatial resolution. This three-instrument PACE mission has the following multiple scientific goals:

- Extending key systematic ocean biological, ecological, and biogeochemical climate data records and cloud and aerosol climate data records;

- Making global measurements of ocean color data products that are essential for understanding the global carbon cycle and ocean ecosystem responses to a changing climate;

- Collecting global observations of aerosol and cloud properties, focusing on reducing the largest uncertainties in climate and radiative forcing models of the Earth system; and,

- Improving our understanding of how aerosols influence ocean ecosystems and biogeochemical cycles and how ocean biological and photochemical processes affect the atmosphere. 


\begin{tabular}{|c|c|}
\hline Parameter & Value \\
\hline Mission & Directed, Design To Cost, $\$ 705 \mathrm{M}$ \\
\hline Mission Life & 3 years, Class $C,{ }^{*} 10$ Years Fuel \\
\hline Launch Vehicle & Atlas V, Antares 231, Falcon 9 \\
\hline Launch & 2022 \\
\hline Orbit & $676.5 \mathrm{~km}$, Sun Sync, 1pm MLT AN \\
\hline Instrument Suite & OCI, SPEXOne, HARP-2 \\
\hline Observatory Mass & 1644 kg NTE \\
\hline Observatory Power & 1000 W NTE \\
\hline Coverage - OCI & 2 day global coverage, \\
\hline $\begin{array}{l}\text { Sun Glint Mitigation } \\
\text { OCI }\end{array}$ & $\begin{array}{l}\mathrm{OCl} \text { Boresight Tilted } \\
+/-20^{\circ} \text { off Nadir, Along Track }\end{array}$ \\
\hline Pointing Knowledge & 90 arcseconds 3 -sigma \\
\hline Pointing Control & 613 arcseconds 3-sigma \\
\hline RF Communication & $\begin{array}{l}\text { Ka Direct to Ground Downlink, } 600 \mathrm{Mbps} \\
\text { Complete Data Set }\end{array}$ \\
\hline Data Storage & 7 Orbits \\
\hline $\begin{array}{l}\text { On-Orbit Absolute } \\
\text { Gain, K1, Calibration }\end{array}$ & $\begin{array}{l}\text { Vicarious Calibration - UVNIR } \\
\text { Solar Calibration - SWIR }\end{array}$ \\
\hline $\begin{array}{l}\text { On-Orbit Gain, K2, } \\
\text { Degradation Trending }\end{array}$ & $\begin{array}{l}\text { Bi-Monthly Lunar Calibration } \\
\text { Daily and Monthly Solar Calibration }\end{array}$ \\
\hline
\end{tabular}

Table 1. PACE Mission Overview

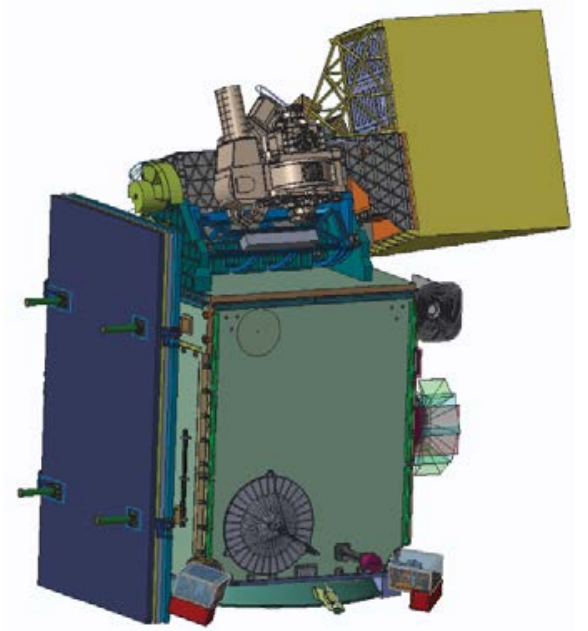

Figure 1. PACE observatory design concept. OCI is at top. Two polarimeters (HARP-2, SPEXone) are located at far right, below the OCI. Folded solar panels are shown at far left. The PACE observatory and OCI are being built by Goddard Space Flight Center.

\subsection{OCI Overview}

PACE's primary sensor, OCI, is a highly advanced optical spectrometer that will be used to measure properties of light over portions of the electromagnetic spectrum. It will enable continuous measurement of light at finer wavelength resolution than previous NASA satellite sensors, extending key system ocean color data records for climate studies.

The color of the ocean is determined by the interaction of sunlight with substances or particles present in seawater such as chlorophyll, a green pigment found in most phytoplankton species. By monitoring global phytoplankton distribution and abundance with unprecedented detail, the OCI will help us to better understand the complex systems that drive ocean ecology.

The OCI is being built at Goddard Space Flight Center. It will consist of a cross-track rotating telescope, thermal radiators, along with half-angle mirror and solar calibration mechanisms. The OCI's tilt will help avoid sun glint and single science detector design will inhibit image striping. 
Its signal-to-noise ratios will rival or exceed previous ocean color instruments.

The OCI will feature:

- Cross track, $360^{\circ}$ continuous rotating telescope

- Two slit grating hyperspectral spectrographs

- (ultraviolet to visible \& visible to near-infrared, NIR)

- Fiber-coupled multiband filter spectrograph

(NIR-to shortwave-infrared)
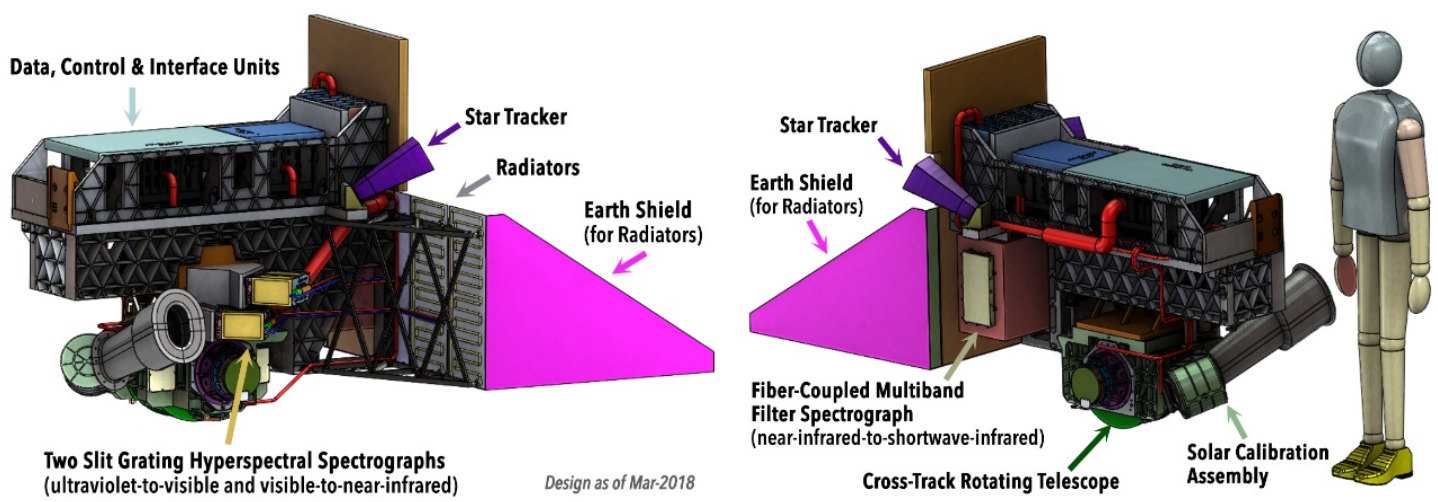

Figure 2. OCI elements

\begin{tabular}{|c|c|}
\hline Instrument Mass & $241 \mathrm{~kg} \mathrm{CBE} \mathrm{,} 283 \mathrm{~kg}$ MEV \\
\hline Instrument Power & 275 W CBE , Orbital Average \\
\hline Thermal System & Passive Cooling with Loop Heat Pipes \\
\hline Mechanisms & $\begin{array}{l}\text { Rotating Telescope Mechanism }(\sim 6 \mathrm{~Hz}) \\
\text { Half Angle Mirror Mechanism }(\sim 3 \mathrm{~Hz}) \\
\text { Solar Calibration Mechanism }(<5000 \mathrm{Cyc})\end{array}$ \\
\hline Deployments & Rotating Telescope Launch Lock \\
\hline Ground Sample Distance & $1050 \mathrm{~m}$ at Nadir \\
\hline Aperture & $90 \mathrm{~mm}$ \\
\hline iFOV & $0.08^{\circ}$ Along Track $\times 1.42^{\circ}$ Cross Track \\
\hline UVNIR System EFL & $0.134 \mathrm{~m}$ \\
\hline UVNIR System f/no & 1.49 \\
\hline Total Field of Regard & $+/-56.5^{\circ}$ \\
\hline Dynamic Range & $S N R$ at $L_{\text {typ }}$, No Saturation at $L_{\max }$ \\
\hline UVNIR Bands (nm) & $342.5 \mathrm{~nm}-887.5 \mathrm{~nm}, 5 \mathrm{~nm}$ Resolution \\
\hline NIRSWIR Bands (nm) & $940,1038,1250,1378,1615,2130,2260$ \\
\hline UVVIS \& VISNIR FPAs & $\begin{array}{l}2 \mathrm{CCDs}, 128 \times 512,26 \text { micron } \\
\text { Integrated } 14 \text { Bit ADC }\end{array}$ \\
\hline NIRSWIR FPAs & $\begin{array}{l}32 \mathrm{MCT} \text { Photodiodes, }<250 \text { micron } \\
\text { Analog output to rem ote SIDECAR ASIC }\end{array}$ \\
\hline $\begin{array}{l}\text { Relative Radiometric } \\
\text { Accuracy }\end{array}$ & $<0.5 \%$ Pre-Launch 1-sigma \\
\hline$S N R @ L_{\text {typ }}$ & $\begin{array}{ll}>1000: 1: & 340-700 \mathrm{~nm} \\
>600: 1: & 700-865 \mathrm{~nm} \\
>50: 1 & 940-2260 \mathrm{~nm}\end{array}$ \\
\hline $\begin{array}{l}\text { On-Board Solar } \\
\text { Calibration Assembly }\end{array}$ & Daily \& Monthly Solar Calibration Targets \\
\hline Orbital Average Data Rate & $12 \mathrm{Mbps}$ up to $40 \mathrm{Mbps}$ \\
\hline
\end{tabular}

Table 2. OCI Overview 


\section{INTEGRATION AND TEST GROUND SUPPORT EQUIPMENT}

The OCI Integration and Test (I\&T) team worked with all the subsystem Product Design Leads (PDLs) and systems engineering to identify any non-flight Ground Support Equipment (GSE) required to support OCI I\&T activities. This GSE falls into two categories; Standard GSE, which is typically used regardless of instrument specific requirements, and Instrument Unique GSE. The OCI unique GSE is specific to the type of instrument testing that OCI requires.

The following are the categories of OCI unique GSE:

- $\quad$ Mechanical GSE (MGSE) (Figure 3)

- $\quad$ Optical GSE (OGSE) (Figure 8, 9 \& 10)

- Thermal GSE (Figure 5)

All OCI test equipment shall be periodically calibrated, and will have certification procedures performed prior to operation per the equipment manufacturer's instructions and GPR 8730.1I, Calibration and Metrology. When EGSE is moved and reconfigured, for example prior to start of environmental testing, an EGSE certification procedure will be performed. All equipment requiring calibration shall have a calibration sticker that indicates that the unit is in calibration if before the calibration due date. Tracking and scheduling the calibration of this equipment will be performed by the OCI I\&T Manager.

To avoid accidental power off of power supplies, all critical power supply On/Off switches shall be covered with a spring-loaded cover. To avoid accidental shorting from external objects of internal power busses, all in-house manufactured or commercial equipment shall have protective covers. All EGSE that interfaces directly with flight equipment shall have a Failure Modes and Effects Analysis (FMEA) performed to ensure that no failure of the EGSE can cause a failure or an overstressed condition of flight hardware. All EGSE connectors mating to flight hardware will be flight connectors.

\section{OCI I\&T GROUND SYSTEM}

The PACE Project and OCI will use Integrated Test and Operations System (ITOS) software as a common I\&T Ground System for testing during OCI I\&T [3]. ITOS provides real-time command and control of the OCI Instrument through all mission phases. The software for ITOS comprises several major subsystems: telemetry and command database,

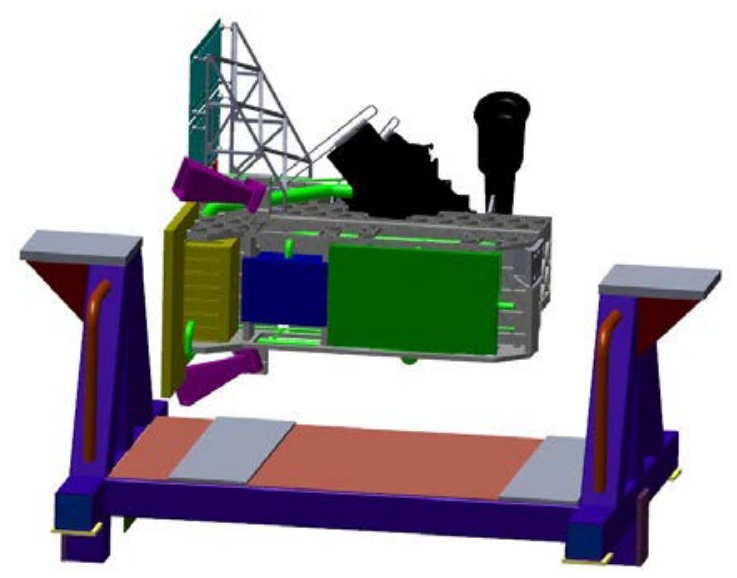

Figure 3. An example of unique MGSE is the OCI Turnover Dolly that will be used to transport OCI between facilities. It rotates 360 Degrees

display pages, and the Systems Test and Operations Language (STOL) interpreter.

This ground system consists of computer workstations and a customized system as the primary interface to the OCI Instrument via the Spacecraft Interface Simulator (SIS) to perform telemetry processing and command encoding.

The OCI Instrument I\&T Local Area Network (LAN) serves as the interface between the ITOS workstations, and other OCI EGSE. It will be utilized during all OCI Instrument powered testing operations prior to observatory level integration.

\subsection{Integrated Test and Operations System (ITOS)}

The Integrated Test and Operations System (ITOS) software is Government Off The Shelf (GOTS) software and provides real time command and control of the spacecraft and associated GSE. ITOS provides a STOL interpreter which can execute procedures and includes various directives for controlling the logical flow of the procedure and for controlling ITOS or other GSE. ITOS provides a graphical user interface for displaying telemetry values, and displays both numeric and graphical elements. Telemetry can be archived and played back, or exported to other tools for analysis. ITOS receives telemetry and transmits commands to the instrument through the SIS using a Transmission Control Protocol/Internet Protocol (TCP/IP) interface. 


\subsection{Front End Data System (FEDS)}

All telemetry data received through the ITOS workstation is stored in ITOS telemetry archives on a local storage array with a minimum size of 500 TB. The file system on the storage array will be shared with other workstations so that any workstation may access and play back telemetry archives. The storage array used by OCI will be a Front End Data System (FEDS).

\subsection{Real-Time Science Processing Workstation}

The Real-Time Science Processing Workstation will provide the capability to receive, process and verify OCI science data packets during OCI I\&T. It will receive science data files through the FEDS during OCI I\&T operations and will be able to display science data in near real-time. It will provide a "Quick Look" at OCI by performing a quick calculation of Time-Of -Flight (TOF) data to assess that testing was successful and OCI can break configuration and move on to the next test. The Science Processing Workstation will receive science data files through the Ocean Data Processing System during OCI I\&T operations and will be able to display TOF data in near real-time.

\subsection{OCI Integrated Trending and Plotting System (ITPS)}

The OCI Integrated Trending and Plotting System (ITPS) workstation receives telemetry from the FEDS and provides the necessary telemetry processing functions to create and maintain an OCI engineering performance archive. The ITPS includes tools to be used by the OCI Test Conductors and systems engineering team to create engineering plots, compute telemetry statistics, and to trend and analyze selected housekeeping telemetry parameters from the telemetry archive. Throughout the OCI I\&T effort, various telemetry points and parameters will be trended and analyzed. The OCI I\&T team will work with the systems and science teams to develop the list of telemetry and parameters which require trending.

\section{OCI INSTRUMENT INTEGRATION AND CALIBRATION}

The start of OCI instrument integration begins with the delivery of OCI components to the OCI I\&T clean room [4], the GSFC Calibration Integration and Alignment Facility (CIAF) shown in Figure 4.
High Bay Area

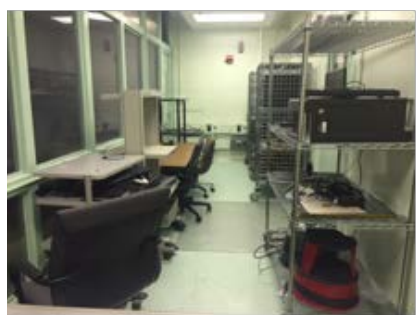

Upper Control Room

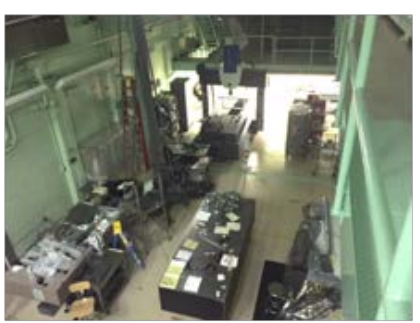

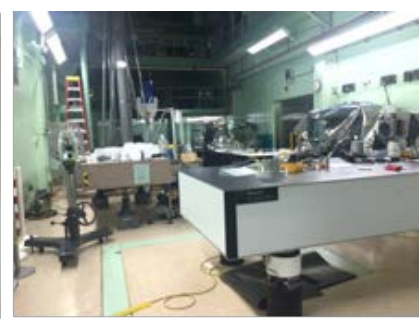

High Bay Area

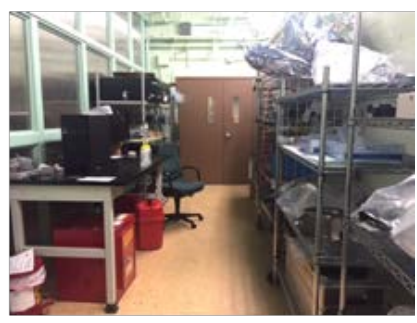

Lower Control Room
Figure 4. GSFC CIAF

The integration and test process begins with receiving the OCI I\&T spacecraft simulator and the Integrated Test and Operations System (ITOS) software. These two systems are integrated together and functionally tested as the OCI Main Electrical Ground Station System (MEGS). Then the MEGS is certified via a procedure which will verify electrically and functionally all interfaces to the OCI instrument.

Once the MEGS is certified, I\&T is ready to receive the Instrument Control and Data Unit (ICDU), the Avionics Interface Plate (AIP) and the Instrument Harness Assembly (IHA). The ICDU is mechanically integrated to the AIP and then electrically integrated to the MEGS via a Safe to Mate (STM) Procedure. Next the Motor Control Electronics (MCE) is received, mechanically integrated to the AIP, and then electrically integrated to the ICDU as per its STM Procedure. After successful functional testing at this level of assembly the system is ready to receive the Main Optics Bench (MOB).

The MOB is mechanically integrated to a Mechanical Ground Support Equipment (MGSE) structure that will be used for transportation and checkout of the Rotating Telescope and Half Angle Mirror. The MOB is electrically integrated to the MCE and then functionally tested. The MOB is the main structure to which the Ultraviolet Near InfraRed Spectrograph (UVNIRS) and Short Wavelength InfraRed Aft Optics Box (SAOB) are mounted. 
Prior to receiving and integration of the UVNIRS and SAOB the Data Acquisition (DAU) unit is received and integrated. The DAU is first mechanically integrated to the AIP and then electrically integrated to the ICDU and MCE. After electrical integration is complete, a functional test is performed to verify data flow from the DAU to the ICDU and the timing between the DAU and MCE. Once the tests are complete then the system is ready for the integration of the UVNIRS and SAOB.

The first detectors to be integrated are the UVNIRS. This unit is mechanically integrated to the MOB and then a series of initial optical alignments, adjustments, and checks are performed. Next the UNVIRS is electrically integrated to the DAU and an initial functional test is performed after which the final optical alignments and adjustments are completed. The SAOB is then mechanically integrated to the MOB and once again initial alignment, adjustments, and checks are performed. The SAOB is then electrically integrated and a functional test is performed. However, full functional testing and final alignment verification cannot be performed for the SAOB until the instrument is under vacuum and the SAOB is at its nominal operating temperature.

At this time the radiator simulators (for ETU) or radiators (for flight) are installed and the instrument is ready to begin the characterization and calibration program which is performed initially at ambient and then completed in vacuum. All of the OCI calibration activities and Thermal Balance/Thermal Vacuum testing will be performed in building 5 OCI Calibration Lab (OCL). The OCL includes a TVAC chamber, reported in Figure 5, a Class 10K Laminar Flow clean tent, reported in Figure 6, as well as 2 black-out clean tents for laser operations. Due to optical stability challenges in I\&T, the chamber is being modified to permit shutting off the cryopumps and running only on Turbopumps which provides a stable optics for the instrument when in vacuum.

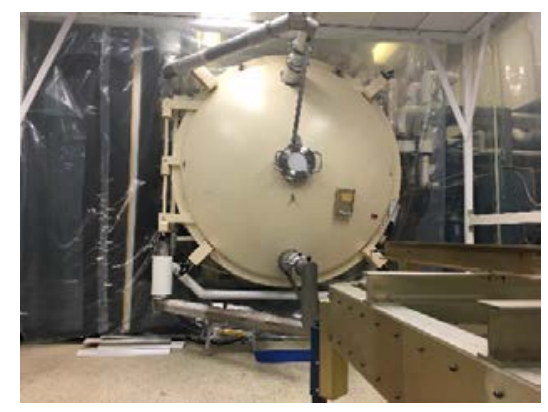

Figure 5. OCL Chamber in building 5

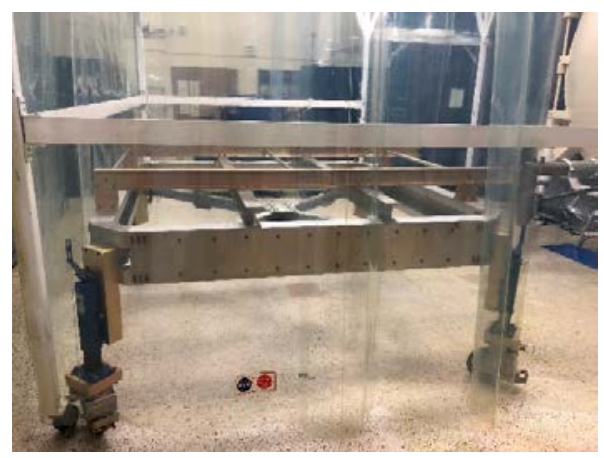

Figure 6. OCL Clean tent in building 5

Features of the OCL include (but are not limited to):

- Fixed / Dedicated / Permanent Clean tent facility

- Secure area with access control

- Class 10K Vertical Laminar Flow Clean tent

- Adequately large enough for the OCI I\&T effort

- 2 black-out tents available for Laser Safety

- Ample area just outside the clean tent for EGSE

- EGSE could possibly remain in place for OCI

EMI/EMC test

- Openings in the clean room wall act as a passthrough for items to be passed into the clean tent.

- An overhead crane

- A large fixed garment changing area

- A nearby large roll-up door

\section{OCI INSTRUMENT CALIBRATION CHALLENGES DURING I\&T}

\subsection{Calibration Sources}

In order for OCI to have the spectral resolution needed for determining the concentrations of the different types of phytoplankton and other constituents of interest, a stable and accurate calibration at every observable wavelength must be performed. To achieve this a wide diversity of calibrated stable narrowband and broadband sources at several different radiance levels and in the Shortwave-Infrared (SWIR) Bands must be provided. Source requirements are reported in Table 3. 


\begin{tabular}{|c|c|c|c|}
\hline OCI Detector & $\begin{array}{c}\text { Wavelength Range } \\
\text { All Wavelengths in }(\mathrm{nm})\end{array}$ & $\begin{array}{c}\text { Calibration Source } \\
\text { Required Bandwidth }\end{array}$ & $\begin{array}{c}\text { Calibration } \\
\text { Required Accuracy }\end{array}$ \\
\hline Hyperspectral Blue & $320-340$ & 0.1 & Best Effort \\
\hline Hyperspectral Blue & $340-400$ & 0.1 & $<=2 \%$ \\
\hline Hyperspectral Blue & $400-605$ & 0.1 & $<=2 \%$ \\
\hline Hyperspectral Red & $600-890$ & 0.1 & $<=2 \%$ \\
\hline SWIR Band & 940 & 1.0 & $<=5 \%$ \\
\hline SWIR Band & 1250 & 1.0 & $<=5 \%$ \\
\hline SWIR Band & 1378 & 1.0 & $<=5 \%$ \\
\hline SWIR Band & 1615 & 1.0 & $<=5 \%$ \\
\hline SWIR Band & 2130 & 1.0 & $<=5 \%$ \\
\hline SWIR Band & 2260 & 1.0 & $<=5 \%$ \\
\hline
\end{tabular}

Table 3. OCI source requirements

The Goddard Laser for Absolute Measurement of Radiance (GLAMR) instrument's importance in providing coverage for the calibration team's source needs is fundamental in the verification of key requirements [5]. The GLAMR team has produced an extremely powerful and adaptable calibration source. GLAMR will provide the OCI calibration team with much of but not all of source scenarios required. GLAMR will not provide broadband light, wavelengths below $340 \mathrm{~nm}$, nor will it provide the highest radiance levels at wavelengths shorter than $680 \mathrm{~nm}$ [6]. There have been several radiance terms invented for the conveyance of expressing the radiance levels that OCI will operate under including Lmax, Lhigh, Ltyp, and Llow [7]. Figure 7 illustrates this and the radiance/wavelength levels that GLAMR can provide. Current GLAMR source data, cannot reach Lmax below 680nm. Broadband source are used to meet dynamic range requirements from $340 \mathrm{~nm}$ to $680 \mathrm{~nm}$.

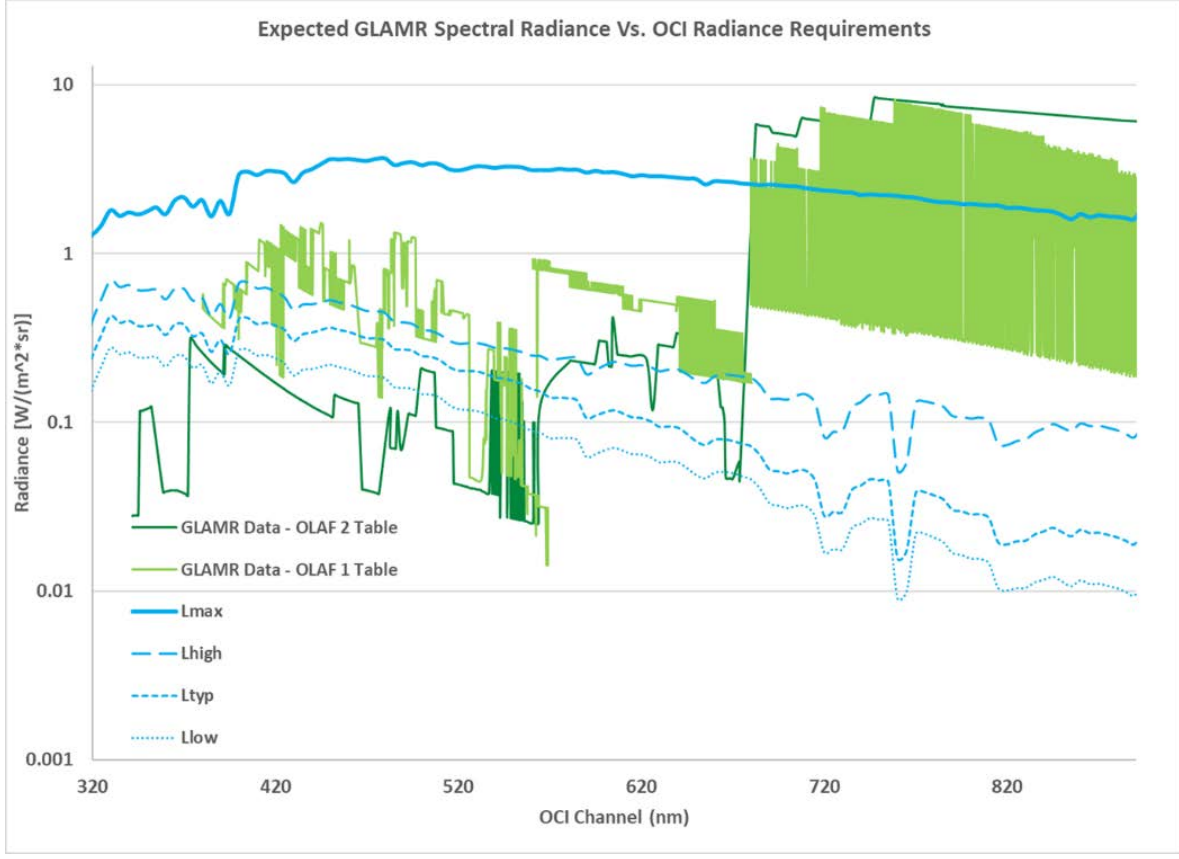

Figure 7. Expected GLAMR Spectral Radiance versus OCI radiance Requirements 
The calibration team will employ a 20” integrating sphere (reported in Figure 8) outfitted with Halogen lamps, an EQ400 Laser-Driven Light-Source (LSDS), Xenon Arc lamps, and calibrated radiometers as the needed broadband calibrated light source that GLAMR cannot provide. In all calibration scenarios where non-coherent and / or non-collimated light is used an integrating sphere is used to create uniform radiance from a source or sources. For wavelengths shorter than 340nm, the extrapolation of discreet source lines (MercuryArgon arc lamps) in combination with narrowband filters may be required for narrowband calibration.

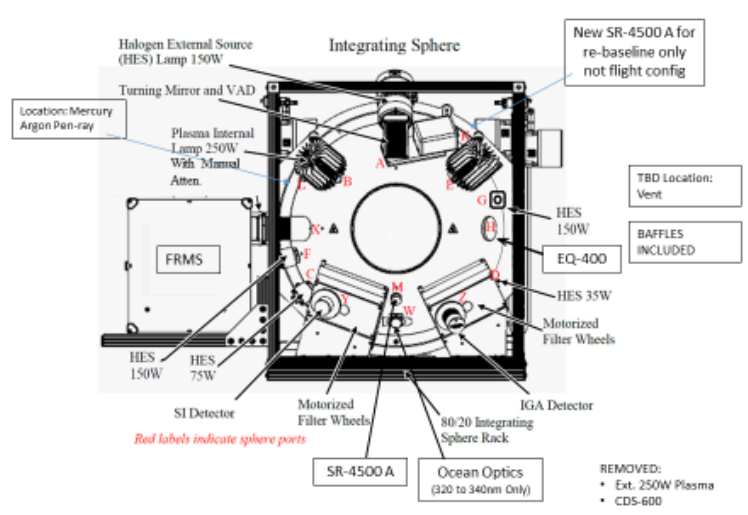

Figure 8. 20" I\&T Integrating Sphere Configuration Diagram

\subsection{Line Spread Function (LSF) Bench}

During flight operations, the OCI instrument will be required to measure the ocean's constituents under varying cloud coverage conditions. The most extreme measurement scenario is measuring the ocean's constituents through a hole in a fully opaque very bright cloud. This requires the calibration team to measure and verify the ability of OCI to separate and measure dark targets when close to or surrounded by very bright objects. The calibration team's answer to achieving this measurement is the Line Spread Function (LSF) bench reported in Figure 9. The LSF bench is an aligned optical projection system that consists of a 2" integrating sphere, various sources, precise masks (normally projected as 1 science pixel), a high-quality 8” off axis parabola (OAP), and a tip-tilt platform. Unlike an integrating sphere, this system will project a bright and collimated precision image into OCI. By looking at the OCI data from this precision incident image, we can determine how well OCI can resolve the features of the image and thus determine how OCI will perform under adverse observing conditions (obstructing clouds).

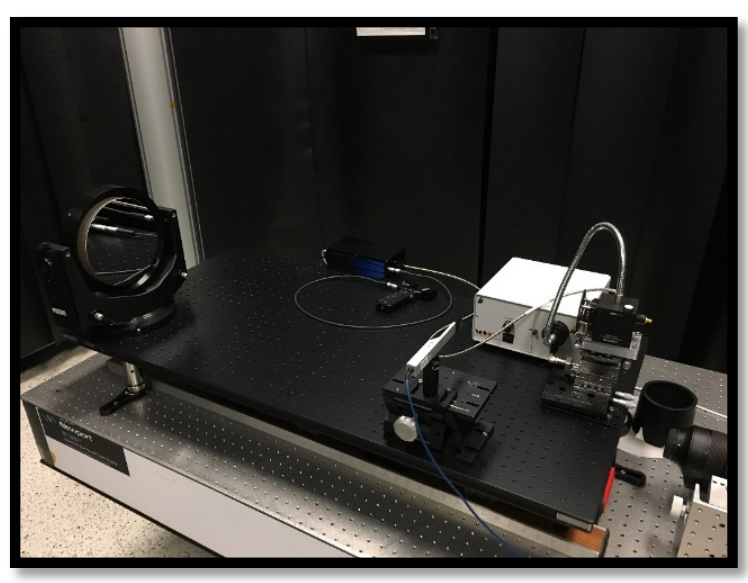

Figure 9. OCI LSF Bench

\subsection{Stray-Light}

Optical instruments normally employ Anti-Reflective (AR) coatings on all of the optical surfaces involved in target observation. This reduces anomalous signal or stray-light within the optical system and increases target signal. It is obviously an advantage to employ AR coatings when attempting to achieve high contrast observations, measure low signal variability, or observe targets with low signal to noise ratios. The OCI instrument examines a wavelength range beginning at the Ultra-Violet and extending to Shortwavelength infrared (SWIR). Unfortunately, AR coatings have effective range limits and no available AR coatings have the extensive wavelength range that the OCI instrument observes. This has resulted in a design that has no AR coatings in the primary optical path of the refractive optics and causes a loss of $5 \%$ to $6 \%$ of the total signal. This along with scattering light inherent within the instrument increases the need for stray-light mitigation and causes the calibration source stray-light tolerances to be extremely limited.

\subsection{Snapshot Mode and Telescope Rotation}

Most optical systems have a fixed primary mirror with downstream optical elements securely fixed in stable focused or optimal positions. The OCI instrument primary mirror assembly or RotationTelescope (RT) rotates at 6 Hertz. At this rotationrate, the RT assembly is subject to a constant centripetal force of approximately $30 \mathrm{Gs}$. The centripetal force during OCI flight operation requires the RT system be assembled in a pre-determined centripetal force influenced optimal position. This presents multiple design, alignment, and calibration challenges. The two different modes of data 
collection during calibration are Normal Mode (6 Hertz RT rotation rate) and Snap-Shot Mode (OCI RT is Fixed). Through OCI data interpretation and optical modeling, the calibration team must understand how OCI calibration data is affected by Snap-Shot Mode operation and determine how accurately the RT system was assembled for Normal Mode operation (Pre-Centripetal force Positioned) through testing at the instrument level.

\subsection{Polarization Sensitivity}

When the Sun's light reflects off the surface of the ocean or an atmospheric cloud, it causes the light to become partially polarized. If polarized light enters either of the OCI's spectral channels, the response of OCI would not be uniform. This non-uniform response or polarization sensitivity would potentially cause large errors of the science outcomes from the data. It has been determined through optical/mathematical modeling that precise alignment of the depolarizer's components is critical to the performance of the depolarizer and therefore critical to OCI's performance. This is why the calibration team must accurately measure the polarization sensitivity of the OCI instrument in the configuration reported in Figure 10.

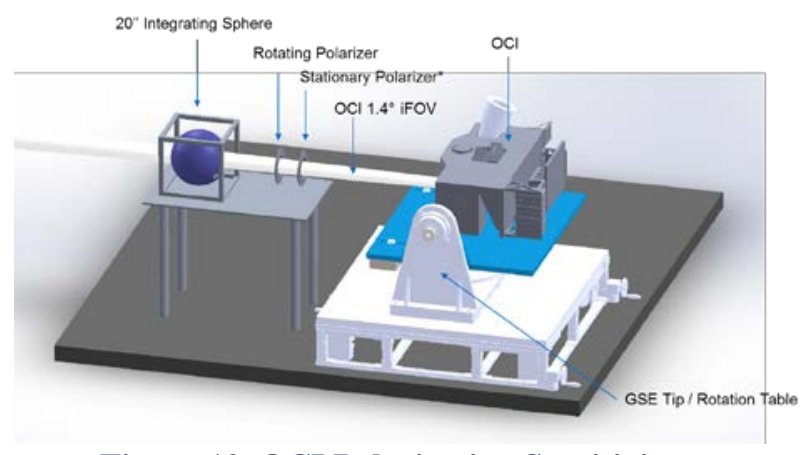

Figure 10. OCI Polarization Sensitivity Measurement Configuration

The longer wavelength SWIR bands detectors must be operated in vacuum. The SWIR detectors are cooled to lower their background signal and therefore to increase their sensitivity. If cooled in air a significant amount of condensation would occur and likely damage their electronics. So, at completion of the calibration process the instrument is in vacuum and a Comprehensive Performance Test (CPT) is run after which the instrument is ready for environmental testing.

\section{OCI INSTRUMENT ENVIRONMENTAL TESTING}

The OCI Environmental Testing phase is kicked-off with the successful completion of the baseline CPT and the OCI Pre-Environmental Review (PER). The OCI Environmental

Tests planned for in order currently include:

- Thermal Balance /Thermal Vacuum / (TB/TV)

- EMI/EMC

- Vibration / Sine Sweep

- Acoustics

- Shock

- $\quad$ Mass Properties / Center of Gravity (CG)

In general, prior to each environmental test, an Aliveness or Limited Performance Test will be performed on the instrument. It will then be configured and readied for the next scheduled environmental test.

\subsection{Thermal Balance/Thermal Vacuum Test}

The TVAC chamber available to the OCI project is the Optical Calibration Laboratory (OCL) Chamber located in the GSFC Building 5/E062 laboratory. This chamber, whose dimensions are reported in Figure 11, is of sufficient size and capacity for the assembled OCI instrument.

The thermal test setup consists of the chamber shroud, cold plates on auxiliary thermal conditioning units (TCUs) and/or heater plates on PID-controlled heater racks that will be used to simulate the space environment of the instrument. A 3 point Thermal Balance (TB) Test will be performed in vacuum to demonstrate the validity of the thermal design of the OCI Instrument. The overall OCI Thermal Balance/Thermal Vacuum (TB/TV) Test will be separated into three distinct phases. These three phases are: (1) the Thermal Balance test, (2) the Thermal Vacuum Test consisting of four thermal cycles, and (3) the final bake-out of the OCI Instrument to meet outgassing requirements.

The test duration will be based on the time required to complete the CPT of the OCI Instrument. All portions of the CPT will be performed once hot and once cold. The test GSE necessary for TV will be installed at the beginning of TB/TV testing and no chamber break will be required. 


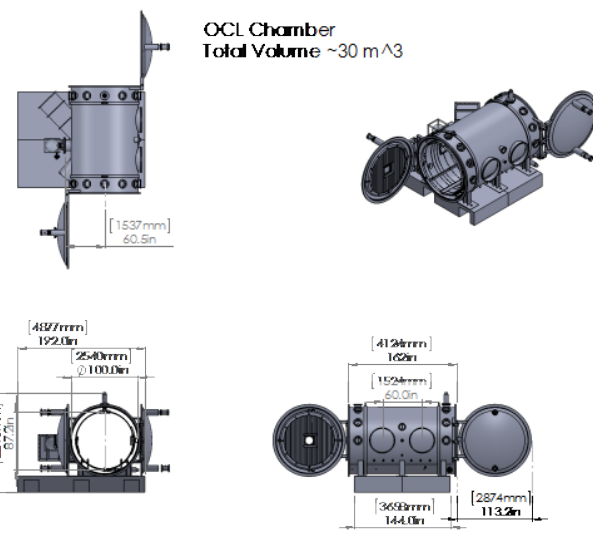

Figure 11. OCL Chamber dimensions

To perform a CPT in the TVAC chamber there are several concerns that have been addressed in order to achieve a calibration required for the OCI instrument. Some of these concerns are: determining a vacuum source window material and mitigating contamination on the window, determining the number of Field of View (FOV)'s OCI can observe through the window, mounting and positioning sources, and determining sources located inside the vacuum envelope. In Figure 12 the 20" CPT integrating sphere is shown attached to the source window port with a light-tight tube.
1.5 FOV Sight to External Integrating Sphere

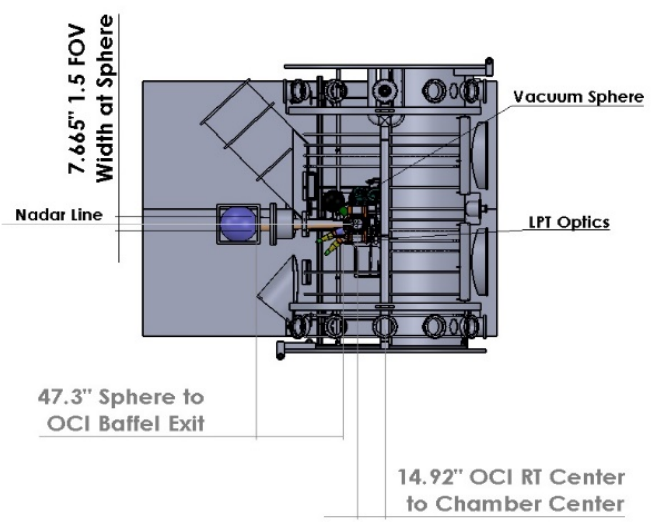

Note: There is $0.335^{\prime \prime}$ of Integrating Sphere Margin for 1.5 FOVs in this Design

Figure 12. 20" CPT integrating sphere attached to the source window port

It is critical that the vacuum source window maintains a high-transmittance that remains stable through the entire wavelength range and during the entire TVAC campaign. The window material chosen is Corning 7979 IR grade fused silica, which has a smooth and continuous $93 \%$ to $94 \%$ transmittance throughout the entire wavelength range without any absorption features. To mitigate contamination depositing onto the window during TVAC operations and changing the window's transmittance a stringent contamination cleaning and control protocol is practiced for the OCL chamber at all times [8]. OCL Chamber Vacuum Source Window Transmittance is shown in Figure 13.

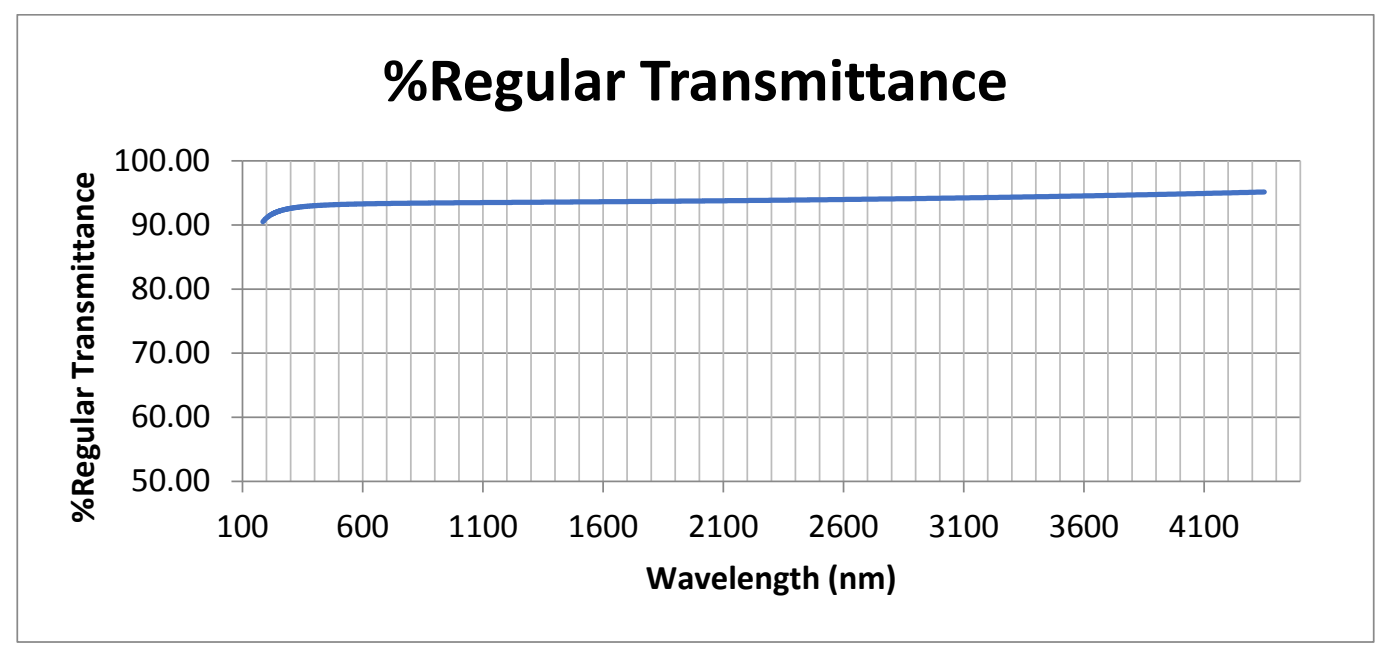

Figure 13. OCL Chamber Vacuum Source Window Transmittance 
In addition to a CPT in the TVAC chamber, a Limited Performance Test (LPT) must be performed and it will be repeated several times during I\&T at both the instrument level and at the spacecraft level. The source used for the LPT is a 12" vacuum compatible integrating sphere equipped with a broadband internal halogen lamp, external fiberoptically connected narrowband sources, and external fiber-optically connected radiometers and narrowband sources. The 12 " vacuum compatible integrating sphere is shown in Figure 14 and it will follow OCI until is delivered for launch.

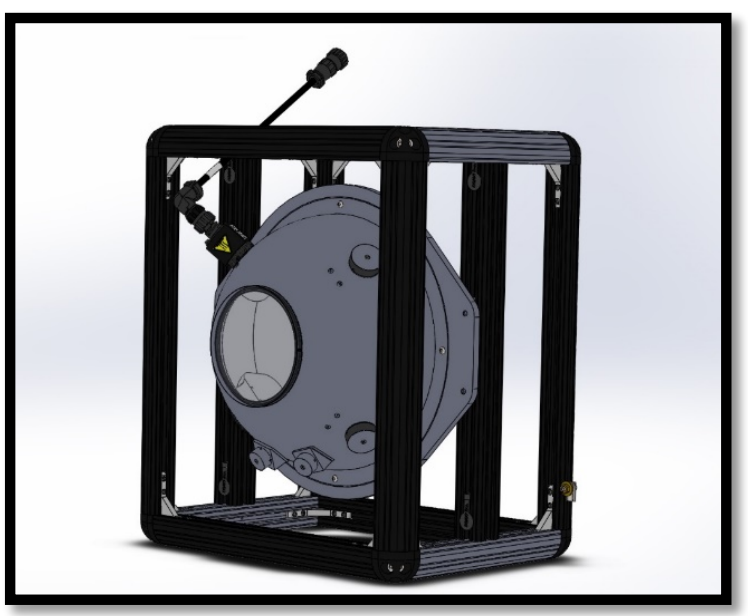

Figure 14. 12" Vacuum Integrating Sphere with Halogen Lamp and 4.5" View Port

After successful completion of Thermal Balance/Thermal Vacuum test the instrument and the MEG are moved from the OCL to building 7/10 complex for EMI, vibration, shock, and acoustic testing. The instrument is operated in several different modes during EMI testing, after each axis of vibration testing, shock, and acoustics testing.

\subsection{EMC/EMI}

Electromagnetic compatibility (EMC) tests will be conducted to ensure that the OCI Instrument does not generate electromagnetic interference (EMI) that will adversely affect the safety and operation of its own systems, the PACE Spacecraft, or the launch vehicle. It will also ensure that the OCI Instrument is not susceptible to EMI that it may be exposed to during its mission life. For radiated and conducted emissions testing, the instrument will be powered and configured in its "noisiest" mode, and emissions will be measured verifying they are within specified limits. For susceptibility testing, the instrument will be powered and configured such that it is in its "quiet" or most sensitive mode. The instrument will then be exposed to various levels of EMI over a range of frequencies. The OCI Instrument level radiated and conducted emissions testing will be performed in the large EMI/EMC test facility with a Class 10K test enclosure shown in Figure 15. OCI personnel and GSE will be located just outside the facility in the connected staging area.

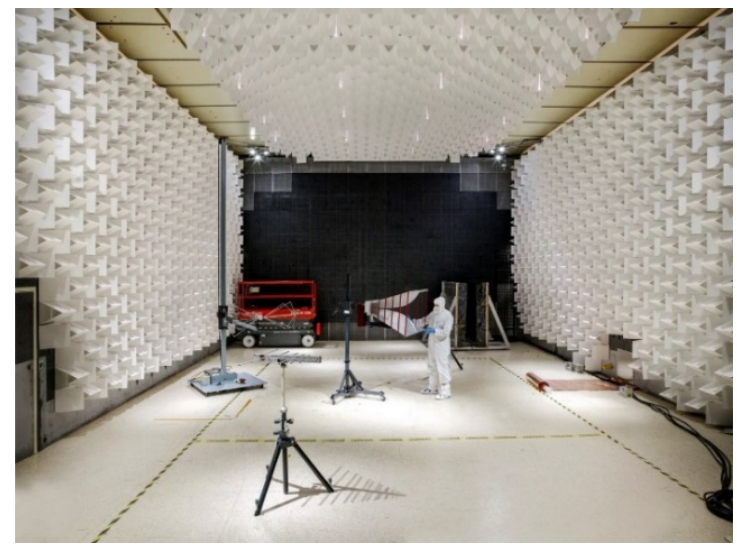

Figure 15. GSFC Large EMC/EMI Facility

\subsection{Vibration Test}

The OCI instrument will use the GSFC Building 7 Vibration Test Facilities to conduct a 3-axis vibration test on the flight unit. The test campaign will utilize the T4000 shaker systems shown in Figure 16. The Vibration Test Cell is a class 100,000 clean room, therefore extra contamination control precautions, including leaving the instrument bagged, will be observed while in this facility and during the vibration tests. The "full-up" OCI Instrument will be subject to a three-axis sine sweep vibration test. The objective of this test is to determine its resonant frequencies, to observe its behavior, and to verify the integrity and workmanship for all mechanical and electrical assemblies on the instrument. The OCI Instrument will be vibrated to specific limits, along one axis at a time, at specific frequencies. During vibration, the instrument will be unpowered and configured in its launch configuration. For this test, the instrument will be mated to an adapter plate that is used to interface the instrument to the vibration table. For the first axis tested, the adapter plate will be pre-installed on the vibration table and the instrument will be lifted onto the vibration cell and mated to it. 


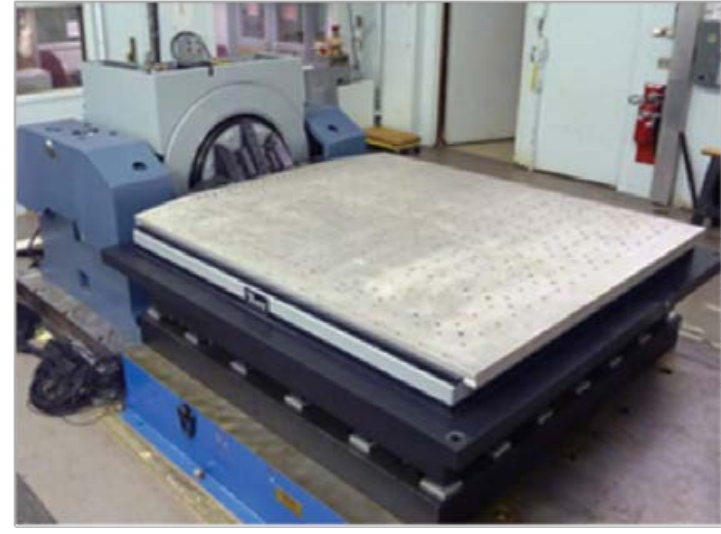

Figure 16. GSFC T4000 Shaker

\subsection{Acoustic Test}

The OCI instrument will use the GSFC Building 10 Acoustic Facility, shown in Figure 17, to conduct an acoustic test on the flight unit. The Acoustic Test Cell is a class 100,000 clean room, therefore extra contamination control precautions, including leaving the instrument bagged, will be observed while in this facility and during the acoustics test. Entry into the Acoustic Test Cell facility is from the GSFC Building 10 High-Bay. OCI will be positioned in the acoustics cell on the Instrument Turn-over Dolly. This test will demonstrate that the instrument will perform without degradation when exposed to an acoustic environment representative of that encountered during launch. The specific acoustic noise levels will be based on project requirements and the selected launch vehicle. During acoustic testing, OCI will be unpowered and configured in its launch configuration.

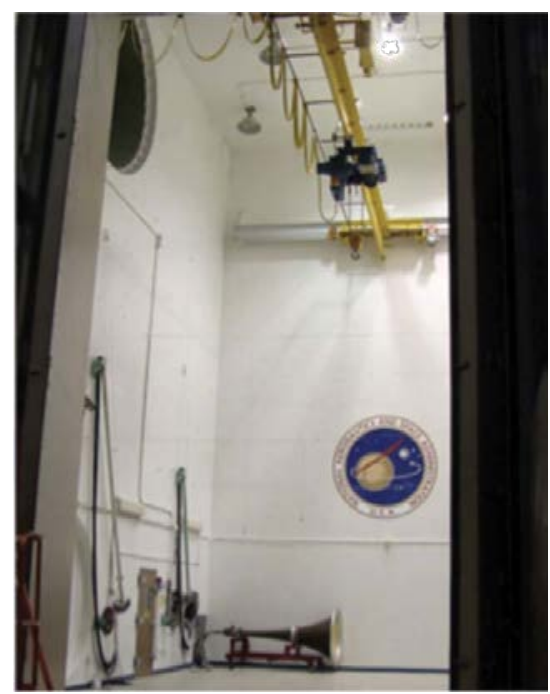

Fig 17. GSFC Acoustic facility

\subsection{Mass Properties and CG Measurement}

The OCI instrument will use the GSFC Mass Properties Measurement Facility (MPMF) to conduct its mass properties test. The MPMF, Figure 18, consists of an air bearing measurement table and scale platform. It can be relocated to the clean room where the instrument is located. An adaptor plate will be provided which will be used to mount OCI to the Miller Table. The test consists of an instrument weight measurement, a two axis center of gravity measurement, and a Moment of Inertia (MOI) measurement about the vertical axis. The OCI instrument will be in near flight configuration and will be unpowered for this test. The Instrument will be un-cabled from any GSE and will be electrically grounded through the test fixture.

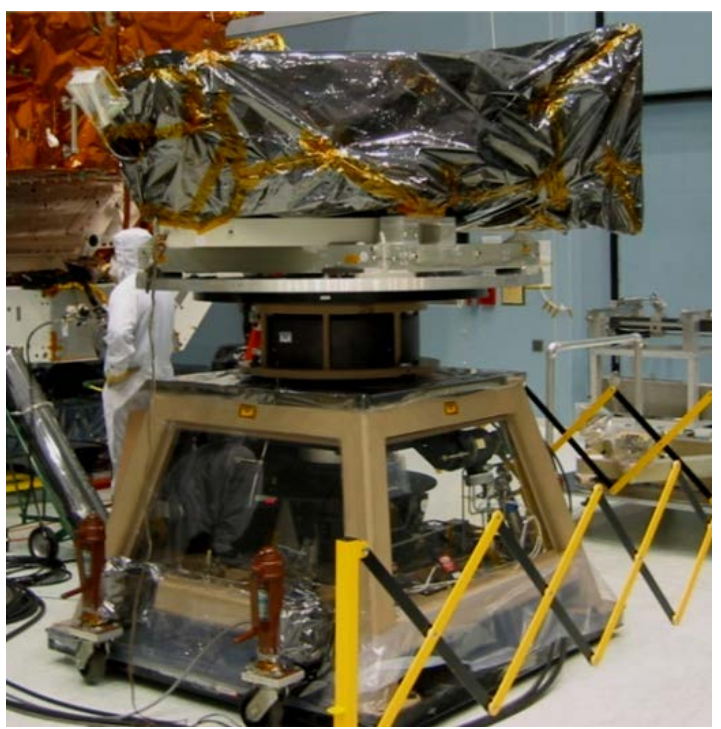

Figure 18. GSFC Mass Properties Measurement Facility

At the successful completion of the OCI I\&T program at GSFC, all required GSE, tools, equipment, documentation, and supplies will be packed into their respective shipping containers and will be moved to the staging area for transport to the PACE I\&T Site. OCI will be configured, cleaned, double bagged and transported to the PACE I\&T site on the OCI turn-over dolly. Most of the packing and loading will occur in the OCL. The OCI Shipping Staging Area will also be in the OCL. 


\section{OCI INSTRUMENT DELIVERY AND OBSERVATORY I\&T ACTIVITIES}

Upon delivery to the Observatory I\&T facility at GSFC, OCI will be unpacked and moved into place along with its GSE to the Observatory clean room. It will be inspected and configured for Acceptance Testing which includes executing a CPT. On completion of this OCI CPT test, the OCI Instrument can be integrated to the PACE observatory. It will then be fully tested with the PACE Observatory and ready for the PACE Environmental Test Program when all of PACEs instruments have been integrated and an Observatory CPT has been performed. OCI acceptance testing completion drives the formal review and handover of OCI to the PACE I\&T team. After successful completion of acceptance testing, final Multi-Layer Insulation (MLI) installation, closeout and cleaning will be performed. The OCI team will maintain responsibility for testing OCI during instrument CPT. At the conclusion of Observatory I\&T, the OCI team will work closely with the PACE I\&T team to ensure that OCI is properly configured and readied for shipment to the launch site. All required OCI GSE will also be configured, packed, and readied for shipment to the PACE launch site.

\section{OCI INSTRUMENT LAUNCH SITE ACTIVITIES}

The PACE Observatory I\&T activities at the Launch Site are primarily the responsibility of the Spacecraft Vendor. The OCI team will work closely with the PACE Observatory I\&T Manager planning launch site activities that can include:

- $\quad$ Support OCI Instrument GSE unpacking, inspection, setup and checkout

- $\quad$ Perform an Instrument CPT and support any required Mission Simulation, Mission Readiness Tests (MRTs), and/or any End-toEnd tests

- $\quad$ Support closeout including MLI of OCI Instrument, including Red Tag/Green Tag closeouts, and final cleaning/inspection

- $\quad$ Support Launch Readiness Review (LRR) and launch operations as required

- $\quad$ Support packing and the return of OCI Instrument GSE back to the GSFC

\section{SUMMARY}

Plans, resources and facilities are in place to assure a successful OCI I\&T campaign. The I\&T plan and the verification matrix are in compliance with the latest requirements documents. Major deliverables are well understood. The baseline plan is to test as OCI will fly. As the test program matures the team may encounter tests that cannot be done in a flight like configuration. The OCI Team will flag those tests that do not meet the test as you fly criteria and will report out on those at the specific reviews. At this time the team does not see any testing that will be performed outside the "as it will be flown" criteria.

Available resources are sufficient to execute the plan. It takes time and effort to integrate members of a team from different disciplines or subsystems. The OCI Integration and Test (I\&T) Manager will also integrate people through planning and coordination of I\&T activities, which will result in increased team dynamics, maximized system testing performances, on-time delivery, and reduced budget over-runs.

Key Considerations for OCI Flight Hardware Safety are: Electrostatic Discharge Protection, Contamination Control, and Laser Safety. OCI personnel will receive the proper training.

All OCI Assembly, Integration and Test Activities will be performed at GSFC facilities. The GSFC facilities provide environmental test capability that ensures flight systems will withstand the launch and will operate properly in space environment. The GSFC Environmental Test and Integration Facilities are one of the most complete and comprehensive complexes within the United States Government.

\section{REFERENCES}

[1] NASA's Plan for a Climate-Centric Architecture for Earth Observations and Applications from Space June 2010

[2] Earth Science and Applications from Space National Imperatives for the Next Decade and Beyond (2007) Consensus Study Report

[3] OCI-INT-PLAN-0005, Revision A Integration and Test Plan Prepared by: David Sohl, NASA Goddard Space Flight Center

[4] OCI-INT-PLAN-0088, Revision -Ocean Color Instrument Integration and Test System Implementation Plan Prepared by: David Sohl NASA Goddard Space Flight Center 
[5] Spectral Testing of the Landsat-9 OLI-2 Instrument using the Goddard Laser Absolute Measurement of Radiance (GLAMR) Julia A. Barsi [a], Joel McCorkel [b], Brendan McAndrew [b], Barbara Zukowski [c], Timothy Shuman [d], Shaida Johnston [e], Brian Markham [b] [a] SSAI, NASA/GSFC Biospheric Sciences Laboratory, Greenbelt, MD, USA 20771; [b] NASA/GSFC Biospheric Sciences Laboratory, Greenbelt, MD, USA 20771; [c] Ball Aerospace; [d] Fibertek; [e] The Aerospace Corporation

[6] Goddard Laser for Absolute Measurement of Radiance for Instrument Calibration in the Ultraviolet to Short Wave Infrared

Brendan McAndrew[a], Joel McCorkel[a], Timothy Shuman[b], Barbara Zukowski[c], Aboubakar Traore[d], Michael Rodriguez[e], Steven Brown[f], and John Woodward[f]

[a]NASA Goddard Space Flight Center, Greenbelt, MD, USA 20771

[b]Fibertek, Herndon, VA, USA 20171

[c]Ball Aerospace, Lanham, MD, USA 20706

[d]NASA Langley Research Center, Hampton, VA, USA 23666

[e] Sigma Space Corporation, Lanham, MD, USA 20706

[f] National Institute of Standards and Technology, Gaithersburg, MD, USA 20899

[7] PACE-SYS-REQ-0004, Revision C OCI Level 3 Requirements Document Prepared by Eric Gorman, NASA Goddard Space Flight Center

[8] OCI-SYS-PLAN-0043, Revision - OCI Contamination Control Plan Prepared by: Kevin Novo-Gradac NASA Goddard Space Flight Center

\section{BIOGRAPHY}

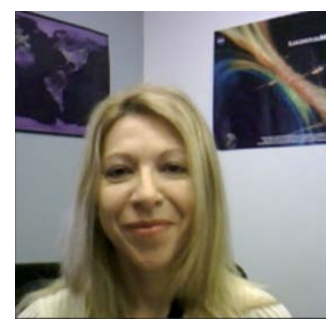

Susanna Petro has more than 30 years of experience in spacecraft and instrument systems design, test, and launch. She currently works as Staff Engineer in the Flight Systems Integration and Test Branch at the Goddard Spaceflight Center. She previously worked at the GOES-R Project supporting the Space Environment In-Situ Suite instruments for technical design, calibration and testing. Before that, she worked at the Johnson Space Center with the Electromagnetic Interference/Compatibility Test \& Analysis Group. She obtained a doctor's degree in Experimental Nuclear Physics from the University of Rome, Italy La Sapienza.

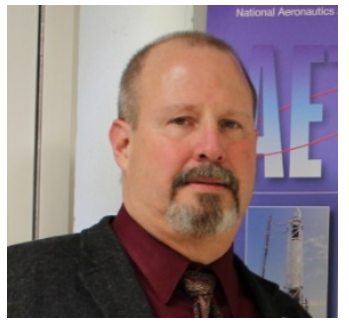

David Sohl has 39 years of experience in aerospace. He earned his Bachelor's degree in Electrical Engineering from the Johns Hopkins Evening College. He is at Goddard Space Flight Center since 1994. He has served as lead electrical design engineer, Integration and Test Manager for the Burst alert Telescope for the Neil Gehrels Swift Observatory, Instrument Manager for the Micro Shutter Development for the Near Infrared Spectrometer on the James Webb Space Telescope, Head of the Instrument Electronics Development Branch, and now Senior Integration and Test Engineer for the Ocean Color Instrument.

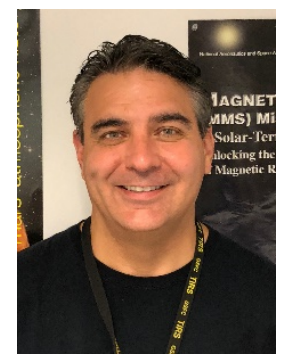

George Hilton is a Senior Engineer and Researcher with twenty five years of experience at NASA Goddard. He is involved in the development of technological advances and meeting engineering challenges in the development, fabrication, testing, and assembly of ground-based and space-based science instruments. He received a B.A. in Mechanical Engineering and a MS in Materials Science Engineering from University of Maryland at College Park.

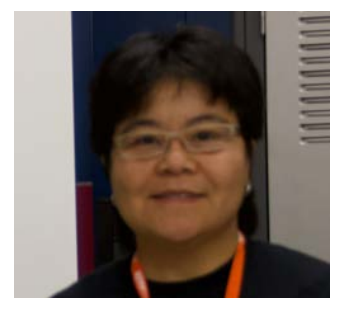

Mellina Espiritu is a Space Simulation Test Engineer and Environmental Project Engineer at Goddard Spaceflight Center. As a lead test engineer of the Environmental Test Engineering and Integration Branch since 2000, she has led the space simulation testing campaign from component to observatory level of several missions, including Lunar Reconnaissance Orbiter (LRO), Thermal Infrared Sensor (TIRS), and Global Precipitation Measurement (GPM), and is currently working on OCI and TIRS-2. She has a BS in Mechanical Engineering from New York University/Polytechnic and an MS in Space Systems from Florida Institute of Technology. 